D.T. Wickramasinghe, L. Ferrario, and G.V. Bicknell, eds.

\title{
Binary Star Formation in Disc-Disc Encounters
}

\author{
S.J. Watkins, A.S. Bhattal, N. Francis, A.P. Whitworth \\ Department of Physics \& Astronomy, University of Wales College of \\ Cardiff, P.O. Box 913, Cardiff CF2 3YB, Wales, U.K.
}

\begin{abstract}
We present results of 3-D self-gravitating hydrodynamical calculations of the encounter between two protostellar discs. We find that the interaction leads to fragmentation of the discs and the formation of new stars and multiple systems.
\end{abstract}

\section{The simulations}

The simulation presented in this poster is one of a number that have been carried out using an SPH code, combined with tree-code gravity, and a realistic treatment of viscosity (Watkins et al. 1996).

We model a coplanar encounter, in which the angular momenta of both discs and the orbit are aligned. The encounter is along a parabolic orbit, and the periastron distance is $1000 \mathrm{AU}$. Each disc has mass $0.5 \mathrm{M}_{\odot}$ and radius $1000 \mathrm{AU}$, and is centred on a star of equal mass; these values correspond to early Class I protostars. The discs are self-gravitating and have an isothermal equation of state at $10 \mathrm{~K}$, becoming adiabatic at densities greater than $10^{-13} \mathrm{~g} \mathrm{~cm}^{-3}$. We use an $\alpha$-viscosity with $\alpha \sim 10^{-3}$ (Shakura \& Sunyaev 1973).

The first four frames of the figure show a time sequence for the encounter. The orbit and discs are rotating clockwise. As the discs collide, material is swept up into a shock layer, which quickly buckles and fragments into two condensations. These condensations merge and fall towards one of the original stars, accreting from its disc as they go, and end up forming a binary with the star. In the meantime the disc around the second star, which is highly perturbed by the encounter, fragments to produce another binary. The remains of the original discs are thrown out in long tidal tails, which surround the stars in a circumbinary-disc-like configuration. The end result of the encounter is two binaries, that are unbound from each other, and which have almost no circumstellar material, but are surrounded by extended circumbinary material. The first binary has masses $0.63 \mathrm{M}_{\odot}$ and $0.26 \mathrm{M}_{\odot}$, with eccentricity 0.2 and periastron $150 \mathrm{AU}$, while the second has masses $0.61 \mathrm{M}_{\odot}$ and $0.035 \mathrm{M}_{\odot}$, eccentricity 0.17 and periastron $88 \mathrm{AU}$. The last two frames of the figure are zoomed out and in respectively from the fourth frame, to show the circumbinary material and the right-hand binary system. 

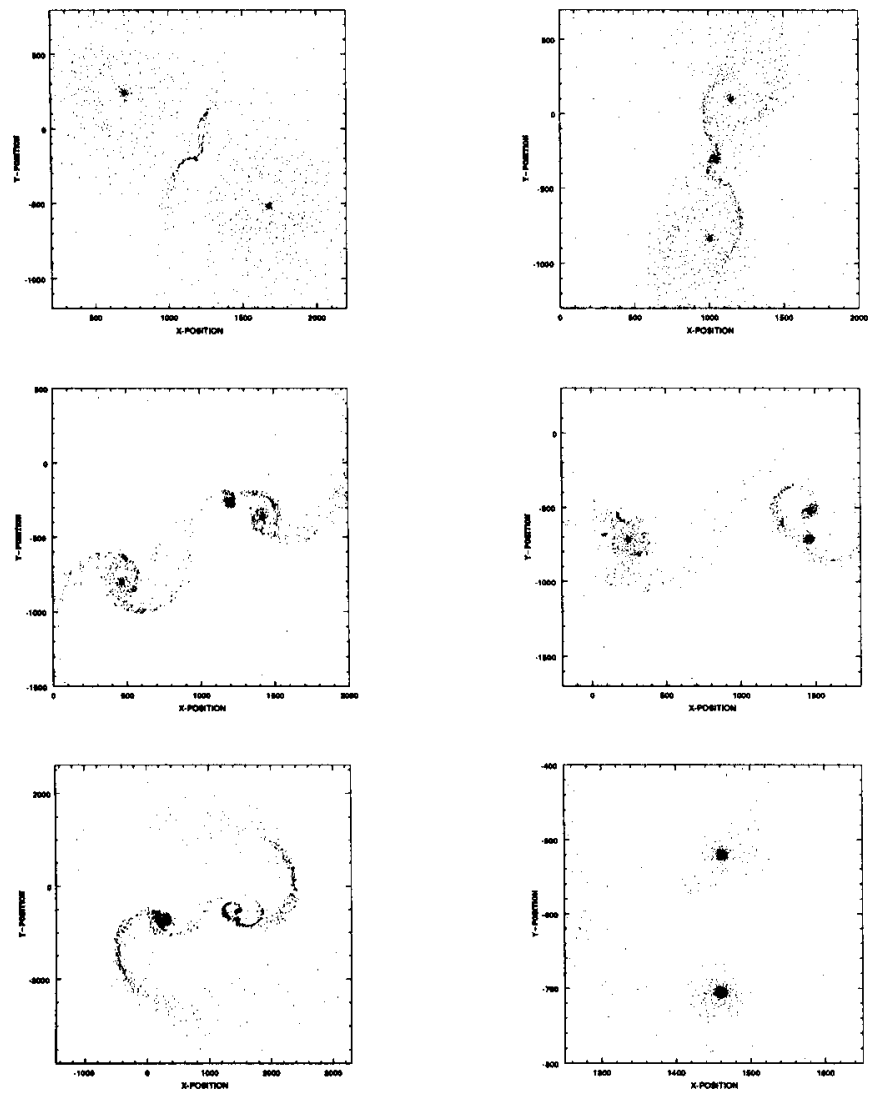

\section{Summary}

The results presented in this poster identify a new mechanism for the formation of close, low-mass binary star systems, in which the interaction between two protostellar discs leads to the formation of a shock layer that then fragments to produce new protostars and binary systems with separations closer than the original system.

\section{References}

Shakura, N.I. \& Sunyaev, R.A., 1973, $A \& A, \mathbf{2 4}, 337$

Watkins, S.J., Bhattal, A.S., Francis, N. \& Whitworth, A.P., 1996, A\&ASS, in press. 